Pacific Journal of Mathematic 


\section{THE MAXIMAL IDEALS OF CERTAIN FUNCTION ALGEBRAS}

\section{RICHARD ARENS}

1. Introduction. In this paper we discover the space of maximal ideals for each Banach algebra of the following concrete type. Select an open subset $G$ of $S$, the compactified complex plane, and let $H(G)$ be the class of complex functions continuous on $S$ and moreover holomorphic on $G$. This is a Banach algebra, and its space of maximal ideals is shown below to be precisely $S$, except in that case in which $G$ is so large as to force $H(G)$ to consist only of constant functions.

Algebras of this type were introduced and studied by J. Wermer [4] and W. Rudin [3]. Wermer pointed out that $H(G)$ need not reduce to the constants even if $S-G$ is required to be (merely) an arc. $\mathrm{He}$ also showed distinct points of $S$ determined distinct maximal ideals. Rudin raised the question as to the space of maximal ideals.

K. M. Hoffman, reporting (April 18, 1958, Symposium on Banach algebras and Harmonic analysis) on work by I. M. Singer and himself jointly, showed that the space of maximal ideals of $H(G)$ is $S$ when $S-G$ has positive upper density at each of its points. On the following day, H. L. Royden's proof was presented in which the same desired conclusion was obtained if $S-G$ has dimension zero. Our technique may be regarded as a refinement of Hoffman and Singer's.

Our methods apply equally easily to more general, although perhaps less interesting, algebras. Let $Z$ be a compact subset of $S$, and let $G$ be an open subset of $Z$. Let $H(G / Z)$ be the functions continuous on $Z$ and holomorphic on $G$. Then $Z$ is the space of maximal ideals, unless the algebra reduces to the constants.

For some algebras in this larger class, the problem can also be solved by an appeal to Mergelyan's theorem [5], namely for those $H(G / Z)$ where $Z \neq S$ and $G$ is the interior of $Z$.

2. An approximation theorem. Let $Z$ be a Borel set in the extended complex plane. Let $G$ be an open set included in $Z$. We denote by $H(G / Z)$ the class of complex-valued functions which are defined, continuous, and bounded on $Z$, and are holomorphic on $G . H(G / Z)$ is evidently a Banach algebra with unit, providing that for each $f \in H(G / Z)$ the norm is defined by

$$
\|f\|=\sup _{\zeta \in Z}|f(\zeta)|
$$

Received May 21, 1958. 
In our study of the maximal ideals of such an algebra, we have been led to an approximation Theorem 2.7 involving functions defined as follows

$$
h(z)=\int \frac{f(\zeta)}{\zeta-z} \mu(d \zeta)
$$

where

(2.1.1) $\mu$ is plane Lebesgue measure restricted to a disc, and then normalized to make the measure of that disc equal to 1.

However, it happens that functions defined as in 2.1 are useful in studying $H(G / Z)$ in still another way. They can sometimes be used to show that there are non-constant elements in $H(G / Z)$. We consider this matter again briefly in $\S 4$. The properties of $h$ needed for this purpose naturally suggest a condition on $\mu$, namely that given by 2.2 .3 (which involves 2.2, 2.1.2). Now it turns out that with no added effort, and very little loss of clarity, a generalization 2.6 of our real objective, 2.7 , can be proved which involves only the quantity $I(\mu)$ of 2.2 .3 , and hence is not confined to the case 2.1.1. It is hoped that some use for the approximation Theorem 2.6 may emerge.

Let $\mu$ be a regular Borel measure in the plane, finite on bounded sets. For $r \geqq 0$ define

$$
m(r)=\sup _{\zeta} \mu\{|z-\zeta|<r\} .
$$

Let $\|\mu\|=\sup _{r} m(r)$; and let

$$
J(\mu)
$$

be the least upper bound, for $0<t_{1}<t_{2}<\cdots<t_{n}$ of the sums

$$
\frac{m\left(t_{1}\right)}{t_{1}}+\frac{m\left(t_{2}\right)-m\left(t_{1}\right)}{t_{2}}+\cdots+\frac{m\left(t_{n}\right)-m\left(t_{n-1}\right)}{t_{n}} .
$$

This is nothing but the Stieltjes integral

$$
\int_{0}^{\infty} r^{-1} d m(r) \text {. }
$$

The class $B$ of measures with which we shall deal are those for which

$$
I(\mu)<\infty .
$$

There are measures in $B$ with 0 -dimensional support (see $\S 4$ ). For our immediate purpose, those given by 2.1.1 are the most important. (Their support is, of course, 2-dimensional). We note the relation of $I(\mu)$ and $\|\mu\|$ in this case.

2.3 Lemma. Let $D$ be a disc of positive radius $\delta$ in the plane. For 
each Borel set $E$ let $\mu(E)$ be the plane measure of $E \cap D$. Then $\delta \cdot I(\mu)=$ $2\|\mu\|$, and $\|\mu\|=\pi \delta^{2}$.

Some properties of 2.1 will now be described.

2.4 Lemma. Let $\mu \in B$, and let $f$ be a bounded Borel-measurable function defined on the plane. Then

$$
h(z)=\int \frac{f(\zeta)}{\zeta-z} \mu(d \zeta)
$$

defines a continuous function on the plane. It is holomorphic on each open set of $\mu$-measure 0 . If $\mu$ has bounded support, $h$ is holomorphic, and 0 , at $\infty$; and $h$ is bounded.

Proof. We treat first the case $f=1$. We will show now that the set functions defined by

$$
F_{z}(E)=\int_{E} \frac{\mu(d \zeta)}{\zeta-z}
$$

are uniformly absolutely continuous (see [2, p. 170]). Suppose $\varepsilon$ is a positive number. Find a number $t$ such that, in the notation of 2.2,

$$
\int_{0}^{t} \frac{1}{r} d m(r)<\frac{\varepsilon}{2} .
$$

Let $\delta=\varepsilon t / 2$. Suppose $\mu(E)<\delta$.

Then

$$
\left|F_{z}(E)\right| \leqq \int_{E} \frac{\mu(d \zeta)}{|\zeta-z|}
$$

We break $E$ into two parts: $E_{1}$, the part on which $|\zeta-z|<t$; and $E_{2}$, the part on which $|\zeta-z| \geqq t$. It is obvious that

$$
\int_{E_{2}|\zeta-z|} \frac{\mu(d \zeta)}{|\zeta-z|}=\frac{\delta}{t}=\frac{\varepsilon}{2}
$$

By breaking $E_{1}$ into concentric annuli, and approximating the integral

$$
\int_{E_{1}} \frac{\mu(d \zeta)}{|\zeta-z|}=I_{1}
$$

by finite sums, it can be shown that

$$
I_{1} \leqq \int_{0}^{t} \frac{1}{r} d m(r) .
$$

This shows that $\left|F_{z}(E)\right|<\varepsilon$ for $\mu(E)<\delta$, independently of $z$, as was to be shown. We may thus apply Proposition $29.6 \mathrm{~s}$ of $[2, \mathrm{p}, 171]$ to conclude that if $z_{n} \rightarrow z$, then

$$
\int_{z}\left|\frac{1}{\zeta-z_{n}}-\zeta-z\right| \mu\left(d_{-}\right) \rightarrow 0
$$


Returning to $h(z)$ as defined by 2.3 , we observe that

$$
\left|h\left(z_{n}\right)-h(z)\right| \leqq\|f\| \int_{z}\left|\frac{1}{\zeta-z_{n}}-\frac{1}{\zeta-z}\right| \mu(d \zeta) .
$$

In view of 2.4.3, the continuity of $h$ is apparent. The holomorphicity at points bounded away from the support of $\mu$ is a similar, actually simpler, proposition. Thus 2.4 is substantially proved.

We now wish to show that with a modified formula we can arrive at a function which is holomorphic wherever $f$ is, even at points of the support of $\mu$.

2.5 Lemma. Let $\mu$ be a measure in $B$ with bounded support. Let $f \in H(G / Z)$. Select any complex number $\lambda,|\lambda| \leqq\|f\|$, and define $f(z)=\lambda$ for $z \notin Z$. Define

$$
h(z)=\int \frac{f(\zeta)-f(z)}{\zeta-z} \mu(d \zeta) .
$$

Then $h \in H(G \mid Z)$, and $\|h\| \leqq 2 I(\mu)\|f\|$. Moreover, $h$ is independent of $\lambda$.

Proof. We write

$$
h_{1}(z)=\int \frac{f(\zeta)}{\zeta-z} \mu(d \zeta), \quad h_{2}(z)=f(z) \int \frac{\mu(d \zeta)}{\zeta-z} .
$$

By 2.4, these are continuous on $Z$. If $f$ is holomorphic at $\infty$, then $h_{1}, h_{2}$ are holomorphic there. Now let $z_{0}$ be a finite point of $G$. Indeed, assume $z_{0}=0$. Then $f$ is holomorphic at 0 . If $f(0)=0$ then $h_{1}, h_{2}$ are both differentiable there. Indeed

$$
\frac{h_{1}(z)-h_{1}(0)}{z}=\int \frac{f(\zeta)}{\zeta} \frac{1}{\zeta-z} \mu(d \zeta)
$$

and

$$
\frac{h_{2}(z)-h_{2}(0)}{z}=\frac{f(z)}{z} \int \frac{\mu(d \zeta)}{\zeta-z} .
$$

Each of these has a limit as $z \rightarrow 0$ because $f(t) / t$ is bounded for all $t$, and continuously definable near 0 . If $f(0) \neq 0$, we replace $f$ by $f-f(0)$. This does not change $h$. Thus $h$ is differentiable at each $z_{0} \in G$.

The main result of this section is as follows:

2.6 THEOREM. Let $z_{0}$ be a point of $Z, z_{0} \neq \infty$, and let $\mu_{1}, \mu_{2}, \cdots$ be a sequence of measures in $B$ such that

(2.6.1) the support of $\mu_{n}$ lies in the $\delta_{n}$-neighborhood of $z_{0}$ where

$$
\delta_{n} \rightarrow 0
$$

and such that for some $M<\infty$, 


$$
\delta_{n} I\left(\mu_{n}\right)<M\left\|\mu_{n}\right\| .
$$

Let $f \in H(G \mid Z)$, and define ${ }^{1}$

$$
h_{n}(z)=\frac{1}{\left\|\mu_{n}\right\|} \int \begin{gathered}
f(\zeta)-f(z) \\
\zeta-z
\end{gathered} \mu_{n}(d \zeta) .
$$

Then

$$
h_{n} \in H(G \mid Z),\left\|h_{n}\right\| \leqq 2 M \delta_{n}^{-1}\|f\|
$$

and

$$
\left\|f-f\left(z_{0}\right)-\left(z-z_{0}\right) h_{n}\right\| \rightarrow 0 .
$$

Proof. It will suffice to treat the case $\left\|\mu_{n}\right\|=1$, and $z_{0}=0$. At first we shall deal with just one $\mu_{n}$, and therefore omit the suffix. We may also confine attention to the case $f\left(z_{0}\right)=0$.

We commence our calculations by observing that

$$
\begin{aligned}
z h(z)-f(z) & =\int\left(z \frac{f(\zeta)-f(z)}{\zeta-z}-f(z)\right) \mu(d \zeta) \\
& =\int \frac{z f(\zeta)-\zeta f(z)}{\zeta-z} \mu(d \zeta) .
\end{aligned}
$$

Let

$$
b(r)=\sup \{|f(z)|:|z| \leqq r\} .
$$

Now suppose $\mu$ is supported by the $\delta$-neighborhood of $z_{0}=0$. Then we may make an estimate

$$
|z h(z)-f(z)| \leqq(|z| b(\delta)+\delta|f(z)|) \int \frac{\mu(d \zeta)}{|\zeta-z|} .
$$

For the integral in 2.6.6 there are two possible estimates:

$$
\int \frac{\mu(d \zeta)}{|\zeta-z|} \leqq \begin{cases}\frac{1}{|z|-\delta} & \text { when }|z|>\delta \\ I(\mu) . & \text { in general. }\end{cases}
$$

The latter of these results from 2.4.2.1 for $t \rightarrow \infty$.

Now let $\varepsilon$ be any positive number. Select a real number $k>1$ such that

$$
2\|f\|<(k-1) \varepsilon .
$$

Let $\mu$ in the preceding discussion be one of the $\mu_{n}$. Then 2.6.6, 2.6.7 hold with $\delta=\delta_{n}$. Consider first a point $z \in Z$ such that $|z| \geqq k \delta_{n}$. We then obtain from 2.6.6, 2.6.7, 2.6.8, that

1 We extend the definition of $f$ to the whole plane, if necessary, by making it have the value $f\left(z_{0}\right)$ everywhere outside of $Z$. 


$$
\begin{aligned}
\left|z h_{n}(z)-f(z)\right| & \leqq \frac{|z|}{|z|-\delta_{n}} b\left(\delta_{n}\right)+\frac{\delta_{n}}{|z|-\delta_{n}}\|f\| \\
& \leqq \frac{k}{k-1} b\left(\delta_{n}\right)+\frac{1}{k-1}\|f\| \\
& <\frac{\varepsilon}{2}+\frac{k}{k-1} b\left(\delta_{n}\right) .
\end{aligned}
$$

Next consider the case $|z|<k \delta_{n}$. Using 2.6.6, 2.6.7 we obtain

$$
\begin{aligned}
\left|z h_{n}(z)-f(z)\right| & \leqq\left[k \delta_{n} b\left(\delta_{n}\right)+\delta_{n} b\left(k \delta_{n}\right)\right] I\left(\mu_{n}\right) \\
& <M\left(k b\left(\delta_{n}\right)+b\left(k \delta_{n}\right)\right) .
\end{aligned}
$$

The numbers $b\left(\delta_{n}\right)$ and $b\left(k \delta_{n}\right)$ tend to 0 since $f$ is continuous at $z_{0}=0$. Therefore there is an $N$ such that $\left\|z h_{n}-f\right\|<\varepsilon$ for $n>N$.

This concludes our proof of 2.6 .

2.7 THEOREM. Let $z_{0}$ be a point of $Z\left(z_{0} \neq \infty\right)$, and let $f \in H(G / Z)$. Then there exist functions $h_{1}, h_{2}, \ldots$ in $H(G / Z)$ such that

$$
\left\|f-f\left(z_{0}\right)-\left(z-z_{0}\right) h_{n}\right\| \rightarrow 0 .
$$

This emerges from the combination of 2.6 and 2.3.

\section{Application to maximal ideals.}

3.1 TheOREM. Let $Z$ be a compact set in the extended complex plane. Let $G$ be an open subset of $Z$. Then the space of maximal ideals of the Banach algebra $H(G \mid Z)$ is naturally homeomorphic to $Z$, provided $H(G \mid Z)$ does not reduce to the constant functions.

Proof. If the set $G$ if void, then the proposition reduces to a case of $[1$, p. 54]. If $G$ is not void, but $Z$ is a proper subset of the extended plane, it is natural to change coordinates so that $Z$ lies in the finite plane. However, the most interesting case, $Z=$ the extended plane, is best treated by having $G$ be a neighborhood of $\infty$. For economy, if not clarity, we perform a conformal transformation, if necessary, to make $G$ (whenever it is not empty) a neighborhood of $\infty$.

Let $F$ be a multiplicative linear functional of $H(G / Z)$. If $G(f)=$ $f(\infty)$ for all $f$ in $H(G / Z)$, then $F$ (or its kernel) corresponds to $\infty$. Having disposed of that unique multiplicative functional, let $F$ be some other one. Then $F\left(f_{1}\right)=1$ for some $f_{1} \in H(G \mid Z)$ such that $f_{1}(\infty)=0$. Then $z f_{1} \in H(G / Z)$. Let $z_{\jmath}=F\left(z f_{1}\right)$. Then $F\left(\left(z-z_{0}\right) h\right)=0$ for each $h \in H(G \mid Z)$, such that $\left(z-z_{0}\right) h \in H(G / Z)$. Because

$$
F\left(\left(z-z_{3}\right) h\right)=F\left(\left(z-z_{0}\right) h\right) F\left(f_{1}\right)=F(h) F\left(\left(z-z_{0}\right) f_{1}\right)=0 .
$$


Now suppose $f \in H(G / Z)$. By 2.7, there exist $h_{n}, r_{n}$ in $H(G / Z)$ such that

$$
f-f\left(z_{0}\right)-\left(z-z_{0}\right) h_{n}=r_{n}
$$

where $\left\|r_{n}\right\| \rightarrow 0$. Hence $F\left(r_{n}\right) \rightarrow 0$. Also, $F\left(\left(z-z_{0}\right) h_{n}\right)=0$. Hence $F\left(f-f\left(z_{0}\right)\right)=0, F(f)=f\left(z_{0}\right)$. Then we say that $F$ corresponds to $z_{0}$.

We have thus shown that to every maximal ideal, or multiplicative linear functional $F$, there corresponds a point $z_{0}$. There might be several such points corresponding to a given $F$. The situation is completely illuminated by a device of Wermer's [4, p. 269] which shows that either $H(G / Z)$ consists only of the constant functions, or some triad of functions separate all pairs of points on $Z$. This completes the proof of 3.1.

We can now acknowledge the relation of our argument to that of Hoffman and Singer. They construct an $h_{n}$, define $r_{n}$ as in 3.2, and show $\left\|r_{n}\right\| \rightarrow 0$; and so forth. Their choice of $h_{n}(z)$,

$$
f(z) \frac{1}{m_{n}} \int_{H_{n}} \frac{d \zeta d \eta}{z-\zeta} \quad(\zeta=\xi+i \eta)
$$

where $E_{n}$ is the intersection of a $\delta_{n}$-disc about $z_{0}$, with $S-G$, and $m_{n}$ is the measure of $m_{n}$, is effective only when $m_{n}>0$ as $\delta_{n} \rightarrow 0$. Hence they assume that $S-G$ has positive upper density at $z_{0}$.

4. Remarks on the dimension of $H(G / Z)$. We return to the question, when does $H(G / Z)$ contain non-constant functions? A sufficient condition is that $S-G$ carry a measure of type $B$, for then 2.4 provides such functions. The formula 2.4 .3 is used in $[3,4]$ for this very purpose, but the measures there employed are absolutely continuous. For this reason it is desirable to point out that there are measures in $B$ that have zero-dimensional support of plane measure zero. An example can be obtained from a well-known function, which increases only at points of the Cantor set. Calling this function $f$, as in [2, p. 49], we form the measure on the line; and then we form the product measure $\mu$ of this measure with itself. It is not hard to see that $I(\mu) \leqq$ $16 \sqrt{2}(1-\lambda)^{-1}$ where $\lambda=\log 4 / \log 3$.

Questions analogous to the above are discussed in $[6,7]$.

\section{REFERENCES}

1. L. H. Loomis, Abstract hnrmonic analysis, D. Van Nostrand, New York, 1953.

2. E. J. McShane, Iniegration, Princeton University Press, Princeton, 1947.

3. Walter Rudin, Algebras of analytic functions, Summary of Lectures and Seminars, Summer Institute on Set-Theoretic Topology, (Amer. Math. Soc.) University of Wisconsin, Mathematics Department, Madison 6 (1955), 137-139.

4. John Wermer, Polynomial approximation on an arc in $C^{3}$, Ann. of Math. Stat., 62 (1955), 269-270. 
5. S. N. Mergelyan, On the representation of functions by series of polynomials on closed sets, Amer. Math. Soc. Translation 85, Providence (R.I.), translated from Doklady Akademii Nauk SSSR (N.S.) 78 (1951), 405-408.

6. L. Ahlfors and A. Beurling, Function-theoretic null seis and conformal invariants, Acta Math., 83, (1950), 101-129.

7. Otto Frostman, Potentiel d'équilibre et capacité . . . (thesis) Lund (1935).

UNIVERSity OF CALIForNiA, LOS ANGELES 


\section{PACIFIC JOURNAL OF MATHEMATICS}

\section{EDITORS}

\section{David Gilbarg}

Stanford University

Stanford, California

\section{R. A. Beaumont}

University of Washington

Seattle 5, Washington

\author{
A. L. Whiteman
}

University of Southern California Los Angeles 7, California

E. G. Straus

University of California

Los Angeles 24, California

\section{ASSOCIATE EDITORS}

\author{
E. F. BECKENBACH \\ C. E. BURGESS \\ M. HALL \\ E. HEWITT
}
A. HORN
V. GANAPATHY IYER
R. D. JAMES
M. S. KNEBELMAN
L. NACHBIN
I. NIVEN
T. G. OSTROM
H. L. ROYDEN

M. M. SCHIFFER

G. SZEKERES

F. WOLF

K. YOSIDA

\section{SUPPORTING INSTITUTIONS}

\author{
UNIVERSITY OF BRITISH COLUMBIA \\ CALIFORNIA INSTITUTE OF TECHNOLOGY \\ UNIVERSITY OF CALIFORNIA \\ MONTANA STATE UNIVERSITY \\ UNIVERSITY OF NEVADA \\ OREGON STATE COLLEGE \\ UNIVERSITY OF OREGON \\ OSAKA UNIVERSITY \\ UNIVERSITY OF SOUTHERN CALIFORNIA
}

\author{
STANFORD UNIVERSITY \\ UNIVERSITY OF TOKYO \\ UNIVERSITY OF UTAH \\ WASHINGTON STATE COLLEGE \\ UNIVERSITY OF WASHINGTON \\ * * * * \\ AMERICAN MATHEMATICAL SOCIETY \\ CALIFORNIA RESEARCH CORPORATION \\ HUGHES AIRCRAFT COMPANY \\ THE RAMO-WOOLDRIDGE CORPORATION
}

Mathematical papers intended for publication in the Pacific Journal of Mathematics should be typewritten (double spaced), and the author should keep a complete copy. Manuscripts may be sent to any one of the four editors. All other communications to the editors should be addressed to the managing editor, E. G. Straus at the University of California, Los Angeles 24, California.

50 reprints per author of each article are furnished free of charge; additional copies may be obtained at cost in multiples of 50 .

The Pacific Journal of Mathematics is published quarterly, in March, June, September, and December. The price per volume (4 numbers) is $\$ 12.00$; single issues, $\$ 3.50$. Back numbers are available. Special price to individual faculty members of supporting institutions and to individual members of the American Mathematical Society: $\$ 4.00$ per volume; single issues, $\$ 1.25$.

Subscriptions, orders for back numbers, and changes of address should be sent to Pacific Journal of Mathematics, 2120 Oxford Street, Berkeley 4, California.

Printed at Kokusai Bunken Insatsusha (International Academic Printing Co., Ltd.), No. 6, 2-chome, Fujimi-cho, Chiyoda-ku, Tokyo, Japan.

PUBLISHED BY PACIFIC JOURNAL OF MATHEMATICS, A NON-PROFIT CORPORATION

The Supporting Institutions listed above contribute to the cost of publication of this Journal, but they are not owners or publishers and have no responsibility for its content or policies. 


\section{Pacific Journal of Mathematics}

\section{Vol. 8, No. $4 \quad$ June, 1958}

Richard Arens, The maximal ideals of certain functions algebras ........ 641

Glen Earl Baxter, An operator identity ........................... 649

Robert James Blattner, Automorphic group representations ........... 665

Steve Jerome Bryant, Isomorphism order for Abelian groups ............ 679

Charles W. Curtis, Modules whose annihilators are direct summands...... 685

Wilbur Eugene Deskins, On the radical of a group algebra ............ 693

Jacob Feldman, Equivalence and perpendicularity of Gaussian

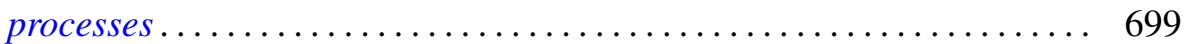

Marion K. Fort, Jr. and G. A. Hedlund, Minimal coverings of pairs by triples....................................... 709

I. S. Gál, On the theory of $(m, n)$-compact topological spaces ......... 721

David Gale and Oliver Gross, A note on polynomial and separable games........................................ 735

Frank Harary, On the number of bi-colored graphs ............... 743

Bruno Harris, Centralizers in Jordan algebras ................... 757

Martin Jurchescu, Modulus of a boundary component ............... 791

Hewitt Kenyon and A. P. Morse, Runs . . . . . . . . . . . . . . . . . . . . . . 811

Burnett C. Meyer and H. D. Sprinkle, Two nonseparable complete metric

spaces defined on $[0,1] \ldots \ldots \ldots \ldots \ldots \ldots \ldots \ldots \ldots \ldots \ldots . \ldots . \ldots . \ldots . \ldots 25$

M. S. Robertson, Cesàro partial sums of harmonic series expansions...... 829

John L. Selfridge and Ernst Gabor Straus, On the determination of numbers by their sums of a fixed order ........................ 847

Annette Sinclair, A general solution for a class of approximation

problems .................................

George Szekeres and Amnon Jakimovski, $(C, \infty)$ and $(H, \infty)$ methods of summation...................................... 867

Hale Trotter, Approximation of semi-groups of operators. ............. 887

L. E. Ward, A fixed point theorem for multi-valued functions ........... 921

Roy Edwin Wild, On the number of lattice points in $x^{t}+y^{t}=n^{t / 2} \ldots \ldots .929$ 